\title{
The Effects of Parental Psychological Control on Non-Suicidal Self-Injury in Chinese Adolescents: The Mediating Effect of Negative Emotions
}

\section{Chao Du, Jianing You ${ }^{*}$, Xiaoling Zheng, Yaxuan Ren and Yongqiang Jiang}

Guang dong Key Laboratory of Mental Health and Cognitive Science, Center for Studies of Psychological Application, School of Psychology, South China Normal University, China

*Corresponding author: Jianing You, Center for Studies of Psychological Applican \& School of Psychology, South China Normal University, Guangzhou 510631, China, E-mail: youjianing@gmail.com

Received date: June 20, 2016; Accepted date: July 01, 2016; Published date: July 08, 2016

Copyright: ( 2016 Du C, et al. This is an open-access article distributed under the terms of the Creative Commons Attribution License, which permits unrestricted use, distribution, and reproduction in any medium, provided the original author and source are credited.

\begin{abstract}
Objective: To explore the relationship between parental psychological control and non-suicidal self-injury (NSSI) and examine whether negative emotions (i.e., anxiety, depression, and stress) mediated this relationship among a Chinese adolescent sample in rural areas.

Methods: A number of 381 Chinese adolescents from a junior high school participated in this study. They aged from 12 to 17 years $(M=13.86, S D=1.08)$. Participants completed questionnaires assessing NSSI, negative emotions and parental psychological control.

Results: We found that negative emotions and parental psychological control were significantly associated with NSSI. What's more, our results showed that negative emotions fully mediated the relationship between parental psychological control and NSSI after controlling for gender and grade.

Conclusion: Parental psychological control as one of the invalidating parenting styles may heighten one's negative emotions, which may then lead to the engagement in NSSI. Implications for the treatment of adolescents with NSSI and suggestions for their parents in the context of the Chinese culture context for rural areas were proposed in the study.
\end{abstract}

Keywords: Non-suicidal self-injury; Parental psychological control; Negative emotions; Adolescents

\section{Introduction}

Non-suicidal self-injury (NSSI), the immediate damage of one's own body without the purpose to die (e.g. cutting, burning, or hitting oneself) become a major public health concern [1]. It is particularly pertinent to adolescents, with the prevalence ranging from $14-56 \%$ [2-4]. NSSI has been included in the newest version of the Diagnostic and Statistical Manual of Mental Disorders (DSM-5) as a new disorder, and was proved to be a potent risk factor for later suicide attempts [5-7], indicating that this problematic behavior deserves more attention. Both intrapersonal and interpersonal risk factors for NSSI have been examined, with most studies focusing on intrapersonal risk factors [8-14]. Within interpersonal risk factors, invalidating family relationships has been paid much attention [15-17]. But the mechanisms underlying the association between invalidating family relationships and NSSI have rarely been studied. The current study aimed to examine the effect of parental psychological control, one aspect of invalidating family relationships, on the engagement of NSSI, as well as the mediating role of negative emotions (i.e., anxiety, depression and stress) in this association among a Chinese adolescent sample in rural areas.

\section{Parental psychological control and NSSI}

Previous theory and research have suggested that parent-child relationship, especially invalidating family environments, may influence the engagement in NSSI in adolescents [18-20]. A great deal of empirical research in this area has identified significant associations between various dimensions of early relationships with parents and NSSI in adolescents and young adulthood. Specifically, childhood physical and sexual abuse, parental control, emotional neglect, disruptions in parent-child bonds, and experiences of separation and loss contributed to later NSSI among adolescent and young adult community samples $[15,16,19-22]$.

Linehan's [18] biosocial theory posited that invalidating family relationships are conceptualized as unsupportive with high levels of negativity and parental control. As reviewed by Rollins and Thomas [23], parental control is further divided into psychological (parental control of the child's psychological world) and behavioral control (control of child's behaviors). Bureau et al. [15] found a positive association between NSSI and both perceived parental behavioral and parental psychological control. Furthermore, Baetens [16] found a significant interaction of parent-reported support and parental behavioral control in relation to NSSI, such that the combination of high control and low support increases for the engagement in NSSI most. However, the underlying mechanism linking parental psychological control and NSSI is still unclear. 
Page 2 of 5

\section{Negative emotions as the mediator}

Parental psychological control had some effects on adolescents' emotional problems. For example, it may lead to negative emotions, including depression, anxiety and stress [24-28]. In addition, some theorists argued that easing negative emotions was the major function of NSSI $[1,29]$.

Additionally, according to Linehan's [18] biosocial theory, adolescents would engage in NSSI to cope with distress (e.g. negative emotions) that is resulted from the invalidating family relationships, indicating that emotional distress may mediates the relationship between invalidating family relationships and NSSI. Some empirical research provided partial support for this argument [8,30-32]. For example, Fruzzetti [8] found that parents' refusal to express their feelings to their children would lead to children's emotion regulation disorders, even NSSI. Beatens [31] also found that depressive symptoms was the mediator of the relationship between lack of family functioning and NSSI among adolescents. However, negative emotions as a possible mechanism to explain the association between parental psychological control and NSSI has been overlooked in previous empirical literatures.

\section{The Present Study}

To partly fill the research gap, the current study aimed to examine the relationship between parental psychological control and NSSI, and to examine whether negative emotions mediated this relationship among a Chinese adolescent sample in rural areas.

\section{Method}

\section{Participants}

Participants were recruited from a junior high school in China. This school is a public school in the countryside. A number of 381 students (213 females) from Grades 7-9 participated, aged from 12 to 17 years $(\mathrm{M}=13.86, \mathrm{SD}=1.08)$. Among them, $83.4 \%$ of the participants were from intact families, $10 \%$ were from single-parent families, $2.6 \%$ were from reconstituted families, and $4.0 \%$ participants didn't report their family structure. The families of more than $3 / 4$ participants $(77.9 \%)$ were located in the countryside.

\section{Procedure}

In this study, all students in Grades 7-9 from the participating school were invited to participate on a voluntary basis. No incentives were given for participation. Because of the cooperation of school authorities, overall student participation rates in Grades 7-9 were close to $94 \%$ for those who were present on the day of data collection. During assessment, the set of questionnaires was group-administered in classrooms of 20-35 students under the supervision of school personnel. At the beginning of each assessment, teachers were required to inform students that they could hand in the questionnaires whenever they wanted and that they did not need to finish all the items if they felt uncomfortable. Adolescents completed questionnaires in their classroom during regular school hours. One or two research assistants were available to provide support if necessary and to ensure independent responding in every classroom. The average time needed to complete the questionnaires was $45 \mathrm{~min}$. Participants were assured of the strict confidentiality of the questionnaires. All testing materials and procedures were approved by the Institutional Review Board.

\section{Measures}

Non-suicidal self-injury. We used the Functional Assessment of selfMutilation Scale (FASM) [33] to measure the method and frequency of NSSI. Participants reported the frequency that they intentionally injured themselves without the intention to die by each of the following 12 ways in the past year: self-cutting, carving, burning, severely scratching, inserting sharp objects to the nail or skin, pulling hair out, biting to injury, erasing skin, eroding skin, bleaching, punching, and banging the head or other parts of the body against the wall. Each of the 12 NSSI items was rated on a 4 -point scale from $1=$ never to $4=$ almost every day. Mean scores of these items were used, with higher scores indicating higher NSSI frequencies. Internal consistencies were adequate $(\alpha=0.80)$ in the current study.

Parental psychological control: The Psychological Control ScaleYouth Self-Report (PCS-YSR) [24] was an 8-item self-report measure. It assesses early adolescents' perceptions of parental psychological control. The scale contains four components: constraining verbal expressions, invalidating feelings, personal attacks, and love withdrawal. Sample items include, "My mother/father often interrupts me" and "My mother/father is a person who is always trying to change how I feel or think about things." Participants was asked to rate each behavior using a 3-point Likert scale ( $1=$ never to $3=$ always). The PCSYSR has satisfactory validity and reliability [34]. Cronbach's alphas of this scale were found to range between 0.72 and 0.86 [25] and was 0.73 for the current study.

Negative emotions: The Chinese version of the short Depression Anxiety Stress Scale (DASS-21) [35] is a 21-item self-report measure of depression, anxiety and stress with each item being scored on 4-point Likert scale $(0=$ do not apply to me at all to $3=$ apply to me very much or most of the time). DASS-21 displayed excellent convergent and discriminant validity, high internal consistency and concurrent validity [36]. In the current study, Cronbach's alphas of the subscales were 0.94 for Depression, 0.87 for Anxiety and 0.91 for Stress.

\section{Results}

\section{Descriptive statistics}

\begin{tabular}{|l|l|l|l|l|l|l|l|}
\hline & & Mean (SD) & $\mathbf{1}$ & $\mathbf{2}$ & $\mathbf{3}$ & $\mathbf{4}$ & $\mathbf{5}$ \\
\hline 1 & Gender & - & - & & & & \\
\hline 2 & Grade & $8.01(0.80)$ & 0.039 & - & & & \\
\hline 3 & $\begin{array}{l}\text { Parental } \\
\text { psychological } \\
\text { control }\end{array}$ & $1.54(0.37)$ & 0.019 & -0.059 & - & & \\
\hline 4 & $\begin{array}{l}\text { Negative } \\
\text { emotions }\end{array}$ & $0.50(0.37)$ & 0.091 & $-0.140^{*}$ & $0.405^{* *}$ & - & \\
\hline 5 & NSSI & $1.17(0.29)$ & $0.128^{*}$ & $-0.109^{*}$ & $0.224^{* *}$ & $0.351^{* *}$ & - \\
\hline
\end{tabular}

Note. NSSI=Non-Suicidal Self-Injury, ${ }^{*} p<0.05,{ }^{* *} p<0.01$

Table 1: Descriptive statistics of study variables and bivariate correlations between them.

Of the sample, 177 participants (48.6\%) reported having engaged in at least one incidence of NSSI. The prevalence of NSSI was $52.8 \%$ in boys and $47.9 \%$ in girls, with no significant gender difference. 
Among the self-injurers, the most common method of NSSI was self-cutting $(46.3 \%)$, followed by severely scratching $(37.8 \%)$ and carving (25.4\%).

The means and standard deviations of all study variables and bivariate correlations between them are presented in Table 1. Parental psychological control was positively associated with negative emotions and NSSI. Meanwhile, negative emotions was positively associated NSSI. Grade was negatively associated with NSSI and negative emotions, with students with higher grades reporting less NSSI and negative emotions. Gender was significantly associated with NSSI. Independent sample $\mathrm{t}$-test showed that boys $(\mathrm{M}=1.21, \mathrm{SD}=0.35)$ engaged in NSSI more frequently than girls $(\mathrm{M}=1.13, \mathrm{SD}=0.19), \mathrm{t}$ $(321.1)=2.594, \mathrm{p}<0.01$.

\section{Primary analyses}

In order to test the mediation model, raw scores of parental psychological control, negative emotions and NSSI were converted into Z-scores. Then analytic steps of testing the mediation effect proposed by Baron and Kenny [37] were employed. Grade and gender entered the model as the control variable.

The first step was to determine if parental psychological control predicted the engagement in NSSI. The result was positive, $b=0.219$, $\mathrm{p}<0.001$. The second step was to determine if parental psychological control predicted negative emotions, the hypothesized mediator. The result also provided support for this relationship $b=0.392, p<0.001$. The third step was to determine the effect of the mediator, negative emotions, on the engagement in NSSI, while controlling for the effect of parental psychological control on NSSI. Results indicated that negative emotions significantly predicted NSSI, $b=0.285, p<0.001$ However, at this step, parental psychological control was no longer related to NSSI significantly, $\mathrm{b}=0.099, \mathrm{p}=0.080$. Therefore, negative emotions fully mediated the relationship between parental psychological control and NSSI (Table 2).

\begin{tabular}{|c|c|c|c|c|c|c|}
\hline & \multicolumn{2}{|l|}{ Step 1} & \multicolumn{2}{|l|}{ Step 2} & \multicolumn{2}{|l|}{ Step 3} \\
\hline & \multicolumn{2}{|l|}{ NSSI } & \multicolumn{2}{|c|}{$\begin{array}{l}\text { Negative } \\
\text { emotions }\end{array}$} & \multicolumn{2}{|l|}{ NSSI } \\
\hline & b & $t$ & $b$ & $t$ & b & $\mathrm{t}$ \\
\hline Grade & -0.133 & $-2.044^{*}$ & -0.149 & $-2.394^{*}$ & -0.105 & -1.613 \\
\hline Gender & 0.252 & $2.410^{*}$ & 0.162 & 1.595 & 0.236 & $2.252^{*}$ \\
\hline $\begin{array}{l}\text { Parental } \\
\text { psychological } \\
\text { control }\end{array}$ & 0.219 & $4.225^{* \star \star}$ & 0.392 & $7.814^{\star \star \star}$ & 0.099 & 1.754 \\
\hline $\begin{array}{l}\text { Negative } \\
\text { emotions }\end{array}$ & & & & & 0.285 & $4.977^{* *}$ \\
\hline $\mathbf{R}^{2}$ & \multicolumn{2}{|l|}{0.076} & \multicolumn{2}{|l|}{0.184} & \multicolumn{2}{|l|}{0.15} \\
\hline $\mathrm{F}$ & \multicolumn{2}{|l|}{$9.501^{* * *}$} & \multicolumn{2}{|c|}{$24.246^{* \star *}$} & \multicolumn{2}{|c|}{$13.872^{* \star *}$} \\
\hline
\end{tabular}

Table 2: The mediating effect of negative emotions on the relationship between parental psychological control and NSSI, ${ }^{*} \mathrm{p}<0.05,{ }^{* *} \mathrm{p}<$ $0.01 .^{* * *} \mathrm{p}<0.001$.

\section{Discussion}

In the present study, we investigated the prevalence of NSSI among a Chinese adolescents sample from a junior secondary school in the rural area. Results showed that $48.6 \%$ of adolescents had engaged in at least one incidence of NSSI. The prevalence seems high, but it was consistent with some previous studies in China [38-40]. Additionally, we found no gender difference on the prevalence of NSSI [41], but boys engaged in NSSI more frequently than girls. This gender difference should be clarified in future studies.

As expected, we found that negative emotions were significantly associated with NSSI. This finding supports previous theories arguing that regulating negative emotions is the major function of NSSI $[1,29]$ and some prior empirical studies. This finding was also consistent with those of previous empirical studies. For example, Xavier and Cunha [42] found depression was significantly predicted NSSI. Other researchers also showed that and anxiety and stress predicted NSSI $[43,44]$. Consistent with Linehan's theory [18], we also found that parental psychological control was significantly associated with NSSI. This result echoes those of previous studies showing that traumatic family experiences (e.g. abused by parents, ignored by parents) were significant risk factors for NSSI $[45,46]$.

The major contribution of this study is that negative emotions fully mediated the relationship between parental psychological control and NSSI, after controlling for gender and grade. Adolescents engaging in NSSI most commonly describe the behavior as a method for coping with negative emotions $[15,41,42]$. Parental psychological control may cause adolescents to be unable to achieve their own needs and desires, which may eventually lead to high depression, low sense of security and belongings $[47,48]$. Moreover, according to the psychodynamic theory of depression and the social expectation model of perfectionism $[49,50]$, lonely adolescents tend to possess negative cognition and attribution styles (e.g. low self-esteem, high self-criticism, and more threaten assessment of general life events) [51]. These negative emotions may then lead adolescents to attempt NSSI [42-44].

The results of this study have some practical implications for the treatment of NSSI. On the one hand, when treating adolescents with NSSI, clinicians should pay more attention to their emotional problems, because emotion dysregulation is likely to be a proximal predictor of NSSI [52]. On the other hand, improving parenting skills of parents and giving psycho-education to parents may also be important for the prevention and intervention of adolescents' NSSI $[28,53]$. As for the specific treatments, cognitive behavioral therapy (CBT) has been indicated as the first choice for anxious youth [54]. Studies comparing family-focused (FCBT) and child-focused (CCBT) interventions demonstrated that both approaches led to ongoing reductions in adolescents negative emotions [55].

Additionally, the association among parental psychological control, negative emotions and NSSI should deserve extra attention in the Chinese culture. It is a very common view among Chinese parents that criticizing children and pointing to them the right way forcibly is good for children [56]. So parents in China exert more parental psychological control on their children [57]. What's more, it has been found that the psychological problems of rural adolescents are more prominent than that of urban adolescents in China [58,59]. Due to the lack of education resources and poor economic situation, the development of adolescents in rural areas is often difficult to meet the expectations of their parents, which may lead to more criticism and psychological control from their parents $[60,61]$. As our results found, 
parental psychological control was not good for adolescents development, and may even cause some psychological problems (e.g. negative emotions) and even the engagement in NSSI. So Chinese parents may learn to ease their psychological controls on children and give children more psychological freedom.

Several limitations should be considered when interpreting findings of the present study. First, we relied solely on participants' self-reports, which may lead to inflation of correlations due to shared method variance. Second, all participants of this study were from a single public school in China, and most of the adolescents were from poor families in rural areas. Thus, whether our results can be generalized to other samples of Chinese adolescents or to adolescents of other countries or cultures still need to be tested in future studies. Third, we used a cross-sectional study design, which did not allow us to examine the temporal relationships between study variables. Fourth, we did not distinguish the effects between mother and father psychological control on sons and daughters. However, previous research showed that the effects may be different. For example, Chen et al. [62] found that fathers criticized their sons more than their daughters. Thus, future research could distinguish between the effects of mother and father psychological control on behaviors of sons and daughters.

In summary, the current study investigated the relationship of parental psychological control and NSSI, and the mediating effect of negative emotions in this relationship. Results demonstrated that parental psychological control may heighten one's negative emotions, which may then lead to the engagement in NSSI. Highlighting the roles of adolescents' own negative emotions and parental psychological control in adolescents' NSSI, these results have clear implications for the prevention and treatment of NSSI.

\section{References}

1. Nock MK (2010) Self-injury. Annu Rev Clin Psychol 6: 339-363.

2. Bjärehed J, Lundh LG (2008) Deliberate self-harm in 14 year old adolescents: How frequent is it and how is it associated with psychopathology, relationship variables and styles of emotional regulation? Cogn Behav Ther 37: 26-37.

3. Hilt LM, Cha CB, Nolen-Hoeksema S (2008) Non-suicidal self-injury in young adolescent girls: Moderators of the distress-function relationship. J Consult Clin Psychol 76: 63-71.

4. Ross S, Heath N (2002) A study of the frequency of self-mutilation in a community sample of adolescents. Journal of Youth \& Adolescents 31: 67-77.

5. You J, Lin MP (2015) Predicting suicide attempts by time-varying frequency of non-suicidal self-injury among Chinese community adolescents. J Consult Clin Psychol 83: 524-533.

6. Guan K, Fox KR, Prinstein MJ (2012) Non-suicidal self-injury as a timeinvariant predictor of adolescent suicide ideation and attempts in a diverse community sample. J Consult Clin Psychol 80: 842-849.

7. Whitlock J, Knox KL (2007) The relationship between self-injurious behavior and suicide in a young adult population. Arch Pediatr Adolesc Med 161: 634-640.

8. Fruzzetti AE, Shenk C, Hoffman PD (2005) Family interaction and the development of borderline personality disorder: A transactional model. Dev Psychopathol 17: 1007-1030.

9. Paivio SC, McCulloch CR (2004) Alexithymia as a mediator between childhood trauma and self-injurious behaviors. Child Abuse Negl 28: 339-354.

10. Zlotnick C, Shea MT, Pearlstein T, Simpson E, Costello E, et al. (1996) The relationship between dissociative symptoms, alexithymia, impulsivity, sexual abuse and self-mutilation. Compr Psychiatry 37: 12-16.
11. Gratz KL, Chapman AL (2007) The role of emotional responding and childhood maltreatment in the development and maintenance of deliberate self-harm among male undergraduates. Psychology of Men \& Masculinity 8: 1-14.

12. Glenn CR, Klonsky ED (2010) A multi-method analysis of impulsivity in non-suicidal self-injury. Personal Disord 1: 67-75.

13. Janis IB, Nock MK (2009) Are self-injurers impulsive? Results from two behavioral laboratory studies. Psychiatry Res 169: 261-267.

14. Stanley B, Sher L, Wilson S, Ekman R, Huang YY, et al. (2010) Nonsuicidal self-injurious behavior, endogenous opioids and monoamine neurotransmitters. J Affect Disord 124: 134-140.

15. Bureau JF, Martin J, Freynet N, Poirier AA, Lafontaine MF, et al. (2010) Perceived dimensions of parenting and non-suicidal self-injury in young adults. J Youth Adolesc 39: 484-494.

16. Baetens I, Claes L, Martin G, Onghena P, Grietens H, et al. (2013) Is nonsuicidal self-injury associated with parenting and family factors? The Journal of Early Adolescence.

17. Spates CR, Samaraweera N, Plaisier B, Souza T, Otsui K (2007) Psychological impact of trauma on developing children and youth. Prim Care 34: 387-405.

18. You J, Leung F (2012) The role of depressive symptoms, family invalidation and behavioral impulsivity in the occurrence and repetition of non-suicidal self-injury in Chinese adolescents: A 2 year follow-up study. J Adolesc 35: 389-395.

19. Linehan MM (1993) Cognitive-behavioral treatment of borderline personality disorder. Current Psychiatry Reports 6: 225-231.

20. Suyemoto KL (1998) The functions of self-mutilation. Clin Psychol Rev 18: 531-554.

21. Van dK, Bessel A (1996) The complexity of adaptation to trauma: Selfregulation, stimulus discrimination and characterological development. Guilford Press.

22. Walsh BW (2006) Treating self-injury: A practical guide. Guilford Press.

23. Conterio K, Lader W, Bloom JK (1998) Bodily harm: The breakthrough healing program for self-injurers. Hyperion Press, New York.

24. Rollins BC, Thomas DL (1979) Parental support, power and control techniques in the socialization of children. The Free Press, Glencoe, NJ.

25. Barber BK (1996) Parental psychological control: Revisiting a neglected construct. Child Dev 67: 3296-3319.

26. Barber BK, Harmon EL (2002) Violating the self: Parental psychological control of children and adolescents. American Psychological Association Press pp: 15-52.

27. Wood JJ, Mcleod BD, Sigman M, Hwang WC, Chu BC (2003) Parenting and childhood anxiety: theory, empirical findings, and future directions. J Child Psychol Psychiatry 44: 134-151.

28. Wang Q, Pomerantz EM, Chen H (2007) The role of parents' control in early adolescents' psychological functioning: a longitudinal investigation in the United States and China. Child Dev 78: 1592-1610.

29. Wijsbroek SAM, Iii WWH, Raaijmakers QAW, Meeus WHJ (2011) The direction of effects between perceived parental behavioral control and psychological control and adolescents' self-reported gad and sad symptoms. Eur Child Adolesc Psychiatry. 20: 361-371.

30. Paivio SC, McCulloch CR (2004) Alexithymia as a mediator between childhood trauma and self-injurious behaviors. Child Abuse Negl 28: 339-354.

31. Chaplo, Shannon D (2015) Harm from harm: extending the construct of moral injury to interpersonal transgressions among emerging adults. Dissertations and Theses - Gradworks.

32. Baetens I, Andrews T, Claes L, Martin G (2015) The association between family functioning and NSSI in adolescence: The mediating role of depressive symptoms. Family Science 6: 1-8.

33. Baetens I, Claes L, Hasking P, Smits D, Grietens H, et al. (2015) The relationship between parental expressed emotions and non-suicidal selfinjury : The mediating roles of self-criticism and depression. Journal of Child \& Family Studies 24: 491-498. 
34. Lloyd EE, Kelley ML, Hope T (1997) Self-mutilation in a community sample of adolescents: Descriptive characteristics and provisional prevalence rates. Poster session at the annual meeting of the Society for Behavioral Medicine.

35. Kincaid C, Jones DJ, Cuellar J, Gonzalez M (2011) Psychological control associated with youth adjustment and risky behavior in African American single mother families. Journal of Child \& Family Studies 20: 102-110.

36. Antony MM, Bieling PJ, Cox BJ, Enns MW, Swinson RP (1998) Psychometric properties of the 42-item and 21-item versions of the depression anxiety stress scales in clinical groups and a community sample. Psychological Assessment 10: 176-181.

37. Chan RC, Xu T, Huang J, Wang Y, Zhao Q, et al. (2012) Extending the utility of the depression anxiety stress scale by examining its psychometric properties in Chinese settings. Psychiatry Res 200: 879-883.

38. Baron RM, Kenny DA (1987) The moderator-mediator variable distinction in social psychological research. Journal of Personality \& Social Psychology 51: 1173-1182.

39. Li Z, Lin DH (2008) Analysis of self-injurious behavior among adolescents -- a case study of Taiyuan in Shanxi. Youth Studies 7: 8-15.

40. Zheng Y (2006) Epidemiological investigation and functional model of self-injury behavior among middle school students in Wuhan. Doctoral dissertation-Central China Normal University.

41. Feng Y (2008) The relationship between self-injury behavior and individual emotional factors or family environmental factors among adolescents. Doctoral dissertation-Central China Normal University.

42. Gratz, Kim L (2003) Risk factors for and functions of deliberate selfharm: An empirical and conceptual review. Clinical Psychology Science \& Practice 10: 192-205.

43. Xavier A, Cunha M, Pinto-Gouveia J (2015) Does self-compassion attenuates the relationship between depressive symptoms and nonsuicidal self-injury in adolescents? The Compassionate Mind Foundation/ International Conference.

44. Kiekens G, Bruffaerts R, Nock MK, Van de Ven M, Witteman C, et al. (2015) Non-suicidal self-injury among Dutch and Belgian adolescents: Personality, stress and coping. Eur Psychiatry 30: 743-749.

45. Kim KL, Cushman GK, Weissman AB, Puzia ME, Wegbreit E, et al. (2015) Behavioral and emotional responses to interpersonal stress: A comparison of adolescents engaged in non-suicidal self-injury to adolescent suicide attempters. Psychiatry Res 228: 899-906.

46. Yates TM, Tracy AJ, Luthar SS (2008) Nonsuicidal self-injury among "privileged" youths: Longitudinal and cross-sectional approaches to developmental process. J Consult Clin Psychol 76: 52-62.

47. Nixon MK, Cloutier PF, Aggarwal S (2002) Affect regulation and addictive aspects of repetitive self-injury in hospitalized adolescents. J Am Acad Child Adolesc Psychiatry 41: 1333-1341.

48. Soenens B, Vansteenkiste M (2010) A theoretical upgrade of the concept of parental psychological control: Proposing new insights on the basis of self-determination theory. Developmental Review 30: 74-99.
49. Lai XF, Zhang W, Bao ZZ, Wang YH, Xiong QL (2014) The relationship between parental psychological control and adolescent depression: A moderated mediation model. Psychological development and education 30: 293-302.

50. Flett GL, Hewitt PL, Oliver JM, Macdonald S (2002) Perfectionism in children and their parents: a developmental analysis. American Psychological Association Press.

51. Hamachek DE (1978) Psychodynamics of normal and neurotic perfectionism. Psychology: A Journal of Human Behavior 15: 27-33.

52. Hawkley LC, Burleson MH, Berntson GG, Cacioppo JT (2003) Loneliness in everyday life: Cardiovascular activity, psychosocial context and health behaviors. J Pers Soc Psychol 85: 105-120.

53. Yurkowski K, Martin J, Levesque C, Bureau JF, Lafontaine MF, et al. (2015) Emotion dysregulation mediates the influence of relationship difficulties on non-suicidal self-injury behavior in young adults. Psychiatry Res 228: 871-878.

54. Connolly SD, Bernstein GA; Work Group on Quality Issues (2007) Practice parameter for the assessment and treatment of children and adolescents with anxiety disorders. J Am Acad Child Adolesc Psychiatry 46: 267-283.

55. Albano AM, Kendall PC (2002) Cognitive behavioral therapy for children and adolescents with anxiety disorders: clinical research advances. Int Rev Psychiatry 14: 129-134.

56. Wood JJ, McLeod BD, Piacentini JC, Sigman M (2009) One-year followup of family versus child CBT for anxiety disorders: Exploring the roles of child age and parental intrusiveness. Child Psychiatry Human Dev 40: 301-316.

57. Li LD, Zheng W, Wang YH, Li DP (2013) The relationship between mother psychological control and adolescents' use of the network: The mediating role of non-adaptive cognition. Psychological Science 36: 411-416.

58. Ahmad, Ikhlas (2009) Mothers' parenting styles as predictors of Palestinian children's peer victimization and aggression. Dissertations and Theses-Gradworks.

59. Shen DL, Ma HX, Bai XJ (2008) The complement of adolescent mental health diathesis questionnaire. Journal of Tianjin Normal University: Social Science Edition 5: 71-75.

60. Duan JL, Che HS, Lv RR (2004) A survey of middle school students' mental health in Beijing. China Doctor in School 18: 302-306.

61. $\mathrm{Li} \mathrm{JH}, \mathrm{He} \mathrm{YL}$ (2009) Research on the relationship between family education environment and mental health of young students. Teaching and management: Theory 3: 49-50.

62. Chen ZJ, Lang Y, Wang L (2011) The relationship between self-injury behavior of junior high school students and parental rearing patterns. Mental health education in primary and secondary schools 16: 19-22. 